\title{
Ordering of Lamellar Block Copolymers on Oxidized Silane Coatings
}

\author{
Nikhila Mahadevapuram ${ }^{\dagger \neq}$, Indranil Mitra ${ }^{\dagger,}$, Shyam Sridhar ${ }^{\ddagger}$, Joseph Strzalkal, \\ and Gila E. Stein ${ }^{\ddagger *}$ \\ $\$$ Department of Chemical and Biomolecular Engineering, University of Houston, Houston, TX \\ $77204-4004$ \\ || X-Ray Science Division, Argonne National Laboratory, Argonne, IL 60439 \\ E-mail: gestein@uh.edu
}

\begin{abstract}
Thin films of lamellar poly(styrene-b-methyl methacrylate) (PS-PMMA) block copolymers are widely investigated for surface patterning. These materials can generate dense arrays of nanoscale lines when the lamellar domains are oriented perpendicular to the substrate. To stabilize this preferred domain orientation, we tuned the substrate surface energy using oxidation of hydrophobic silane coatings. This simple approach is effective for a broad range of PS-PMMA film thicknesses when the oxidation time is optimized, which demonstrates that the substrate coating is energetically neutral with respect to PS and PMMA segments. The lamellar films are characterized by high densities of defects that exhibit a strong dependence on film thickness: in-plane topological defects disrupt the lateral order in ultrathin films, while lamellar domains in thick films can bend and tilt to large misorientation angles. The types and densities of these defects are similar to those observed with other classes of neutral substrate coatings, such as random copolymer brushes, which demonstrates that oxidized silanes can be used to control PS-PMMA self assembly in thin films.
\end{abstract}

*To whom correspondence should be addressed 
$\dagger$ Equally contributing authors.

Keywords: Block copolymer, Thin Film, Lithography, Patterning, Silane, GISAXS

\section{Introduction}

Block copolymers can spontaneously self-assemble into dense arrays of nanoscale domains. In a thin film, these systems have been used for patterning nanowire arrays, ${ }^{1}$ nanoscale sensors, ${ }^{2}$ and nanoscale devices for integrated circuits. ${ }^{3,4}$ Most examples of block copolymer patterning use vertically-oriented lamellar or cylindrical domains to produce nanoline or nanodot templates, respectively. The most common block copolymer chemistry for patterning is the diblock copolymer poly(styrene-b-methyl methacrylate) (PS-PMMA). ${ }^{3,5}$ The advantages of this system include commercial availability, easy pattern development, and simple methods to create "neutral" interfaces that do not preferentially attract the PS or PMMA segments. Neutral interfaces are critical for stabilizing a vertical domain orientation over a broad range of film thickness. ${ }^{6,7}$

Neutral substrate coatings are usually required for PS-PMMA patterning. The most popular coating materials are based on random copolymers of styrene and methyl methacrylate. ${ }^{5,8-15}$ In these systems, the optimal proportion of styrene to methyl methacrylate is determined by the composition of the block copolymer, ${ }^{5,7-10}$ as well as the coating thickness and density. ${ }^{12-15}$ While there are a few types of commercially-available random copolymer coating materials, most are synthesized in a university laboratory to target a specific composition and molecular weight. Furthermore, parameters like coating thickness and density are partially controlled by the deposition process and can be difficult to accurately characterize. Therefore, an easy process that tunes substrate properties with commercial materials and simple instrumentation could be very valuable for block copolymer patterning.

In this manuscript, we report a simple approach to generate neutral interfaces for PS-PMMA lamellae. We employ high temperature processing to suppress the formation of PS wetting layers at the free surface, ${ }^{7,13}$ and we create neutral substrates through oxidation of hydrophobic 
organo-silane coatings. Organo-silanes are commercially-available small molecule hydrocarbons that spontaneously assemble on the native oxide of a clean silicon wafer. ${ }^{16}$ There are several literature studies that used silanes to engineer surface interactions for block copolymer self-assembly. As examples, silane mixtures that mimic the chemistry of each segment can produce a neutral or preferential boundary, ${ }^{17-19}$ and patterned silane coatings can serve as epitaxial templates for directed self-assembly. ${ }^{20}$ The use of silane oxidation to generate neutral coatings is less studied. One work demonstrated the potential of this approach using ultrathin films of PS-PMMA cylindrical and lamellar phases on oxidized hydrophobic silanes. ${ }^{21}$ Herein, we demonstrate that vertical lamellar domains can be stabilized across a broad range of film thicknesses when the oxidation time is optimized, but mixed domain orientations are observed at certain film thicknesses if the oxidation time is too short or too long. Furthermore, using microscopy and x-ray scattering, we demonstrate that lamellar defect structures on oxidized silane coatings are similar to those encountered with the more common neutral polymer coatings. Therefore, we conclude that oxidized silanes can replace other neutral coatings for PS-PMMA patterning.

\section{Experimental Procedures}

Materials: The diblock copolymer used for these studies is a lamellar poly(styrene- $b$-methyl methacrylate) (PS-PMMA) with $53 \mathrm{vol} \% \mathrm{PS}, M_{n}=98 \mathrm{~kg} / \mathrm{mol}$, and $Ð=1.12$. This material was purchased from Polymer Source and independently characterized at the University of Houston with nuclear magnetic resonance spectroscopy and gel permeation chromatography. The equilibrium lamellar periodicity $\left(L_{0}\right)$ for this PS-PMMA copolymer is $46 \mathrm{~nm}$ (measured with grazingincidence small angle $\mathrm{X}$-ray scattering). The segregation strength for this polymer is $\chi N \simeq 45$ and approximately independent of temperature. ${ }^{22}$ Substrates were 3-inch diameter, p-type (100)oriented silicon wafers with standard resistivity and thickness. Self-assembled monolayers were prepared with octyldimethylchlorosilane (ODS), which has a very low vapor pressure of $6.7 \mathrm{kPa}$ at room temperature. ODS was purchased from Gelest. 
Substrate functionalization: Silicon substrates were cleaned with a UVOCS ultraviolet/ozone system (wavelengths of $185-254 \mathrm{~nm}$, and intensity of $2.5 \mathrm{~mW} / \mathrm{cm}^{2}$ ) for 20 mins to destroy organic contamination and grow a thin oxide layer. ODS was vapor deposited on the oxidized silicon using low vacuum and ambient temperature. The deposition time ranged from 48-60 hours. This excessive deposition time generates a thick coating of ODS on the wafer, meaning there is a film on top of the surface-attached monolayer, and the extra material is removed through the following stages: First, the samples were gently sonicated in a 50:50 (vol/vol) mixture of water and chloroform for 5 $\min$. Second, the wafers were gently sonicated in toluene for $2 \mathrm{~min}$. Third, the wafers were gently sonicated in isopropyl alcohol for $1 \mathrm{~min}$. Finally, the wafers were gently sonicated in deionized water for $1 \mathrm{~min}$, and then dried under a nitrogen stream. The thickness of the ODS coating was measured with a J.A. Woollam spectroscopic ellipsometer. Each data set consists of the parameters $\Psi$ and $\Delta$ that describe the change in polarization state of light. These data were modeled with a predetermined refractive index for native oxide and bulk silicon, and the refractive index of the silane was modeled with the Sellmeier relation $n(\lambda)=a+b \lambda^{2} /\left(\lambda^{2}-c^{2}\right)-d \lambda^{2}$, where $\lambda$ is the wavelength of incident light. The oxide thickness was constrained to $1 \mathrm{~nm}$, and the Sellmeier constants and ODS film thickness were adjustable parameters for regression analysis. Typical values of the Sellmeier constants are $a=2.35, b=0.29, c=0.35 \mu \mathrm{m}$, and $d=0.06 \mu \mathrm{m}^{-2}$. The ODS thickness determined with this approach was approximately $1 \mathrm{~nm}$ for all samples, which is consistent with a self-assembled monolayer. The substrate surface energy was tuned by oxidizing ODS in the UVOCS system for up to $60 \mathrm{sec}$.

Surface Energy. The surface energies of functionalized wafers (ODS and oxidized ODS) were calculated from equilibrium contact angle measurements based on Wu's harmonic method. ${ }^{23,24}$ Two liquids were used, diiodomethane (non-polar) and deionized water (polar), and equilibrium contact angles were recorded from at least three points on the substrate using a DataPhysics OCA $15 \mathrm{EC}$ goniometer. 
X-Ray Photoelectron Spectroscopy (XPS): XPS was used to characterize the surface composition of functionalized wafers as a function of oxidation time. Measurements were performed with a Surface Science Instruments model M-probe 301. The samples were illuminated with monoenergetic $\mathrm{Al} \mathrm{K} \mathrm{K}_{\alpha}(1486.6 \mathrm{eV})$ radiation over a $300 \mu \mathrm{m}$ diameter area. Base pressure in the XPS chamber was maintained at $5 \times 10^{-10}$ Torr. The emission angle of electrons was set at $30^{\circ}$ with respect to the sample plane, which samples a depth of 2-3 nm assuming an average attenuation length for electrons equal to that of $\mathrm{Si}(22 \AA)$. The aliphatic carbon $(\mathrm{C}-\mathrm{C}, \mathrm{C}-\mathrm{H})$ at a binding energy of $285 \mathrm{eV}$ (C 1s photoline) was used to calibrate the binding energies.

PS-PMMA Thin Films: Thin films of PS-PMMA block copolymers were prepared on the functionalized silicon surfaces. PS-PMMA was dissolved in toluene at concentrations that ranged from 1-4 wt $\%$, and then solutions were filtered with a $0.2 \mu \mathrm{m}$ Teflon mesh. Films with thickness in the range of $20 \mathrm{~nm}$ to $140 \mathrm{~nm}$ were prepared by spin-casting, and all samples were annealed using a Brewer Science hot plate (with heat shield) for 10 minutes in air at $240{ }^{\circ} \mathrm{C}$. The thickness of PS-PMMA films was measured with a J.A. Woollam spectroscopic ellipsometer. The ellipsometry parameters $\Psi$ and $\Delta$ were modeled with a predetermined refractive index for the native oxide and bulk silicon, while the refractive index of PS-PMMA was modeled with the Cauchy dispersion relation $n(\lambda)=A+B / \lambda^{2}$. The oxide thickness was constrained to $1 \mathrm{~nm}$, so the only adjustable parameters for regression analysis were $A, B$, and film thickness (all positive values). Typical values for the Cauchy constants were $A=1.52$ and $B=0.01$

Microscopy. The nanoscale and microscale structure at the surface of PS-PMMA films was characterized with atomic force microscopy (AFM) and/or scanning electron microscopy (SEM). AFM micrographs were collected with a MultiMode 3 (Veeco) in Tapping Mode using silicon probes with a spring constant of approximately $40 \mathrm{~N} / \mathrm{m}$. Typical parameters for data acquisition were 1.7 $\mathrm{Hz}$ scan frequency, $5 \mu \mathrm{m} \times 5 \mu \mathrm{m}$ scan area, and $512 \times 512$ image resolution. The SEM images were recorded with a FEI XL-30FEG instrument in the secondary electron mode. The parameters used for data acquisition were an accelerating voltage of $5 \mathrm{kV}$, beam current of $95 \mathrm{pA}$, working 
distance of $5 \mathrm{~mm}$, and magnifications ranging from 50-65 kx.

Grazing-Incidence Small Angle X-Ray Scattering (GISAXS). GISAXS experiments were conducted at beam line 8-ID-E at the Advanced Photon Source of Argonne National Laboratory. Samples were placed in a vacuum chamber and illuminated with $7.35 \mathrm{keV}$ radiation at incident angles in the range of $0.2-0.24^{\circ}$; the off-specular scattering was recorded with a Pilatus $1 \mathrm{MF}$ pixel array detector (pixel size $=172 \mu \mathrm{m}$ ) positioned $2175 \mathrm{~mm}$ from the sample. Acquisition times were approximately $10 \mathrm{sec}$ per frame. Each data set is stored as a $981 \times 1043$ 32-bit tiff image with 20-bit dynamic range. All data are displayed as intensity maps $I(2 \Theta, \alpha)$, where $2 \Theta$ and $\alpha_{f}$ denote in-plane and out-of-plane diffraction angles, respectively.

\section{Results and Discussion}

The chemical structure of the oxidized ODS surfaces was evaluated with XPS, and data from survey scans are reported in Figure S1. As expected, the ratio of carbon-to-oxygen decreases with increasing oxidation time. High resolution scans of the carbon edge at each oxidation time are included in the Supporting Information Figure S2; however, these data do not offer any insight into the oxidation products because the line shapes are equivalent within experimental error. The surface energies of oxidized ODS/silicon substrates were determined through measurement of equilibrium liquid contact angles (water and diiodomethane). Contact angles $(\theta)$ are summarized in Fig. 1a as a function of oxidation time, and the corresponding surface energies $(\gamma)$ through Wu's harmonic method ${ }^{23,24}$ are reported in Fig. 1b. The surface energies of PS and PMMA homopolymers are approximately 40 and $44 \mathrm{mN} / \mathrm{m}$, respectively, while their melt surface tensions are both near $37 \mathrm{mN} / \mathrm{m}$ at the annealing temperature of $240^{\circ} \mathrm{C} .{ }^{25,26}$ Therefore, one might anticipate that "neutral" substrates (equal preference for PS and PMMA) are generated from oxidation times in the range of $10-30 \mathrm{sec}$. This corresponds with water contact angles $\left(\theta_{w}\right)$ from $95^{\circ}$ down to $78^{\circ}$. As a reference, the water contact angles on pure PS and PMMA surfaces are $90^{\circ}$ and $70^{\circ}$, respectively.

Thin films of PS-PMMA lamellar copolymers were spin-casted on the oxidized ODS/silicon 


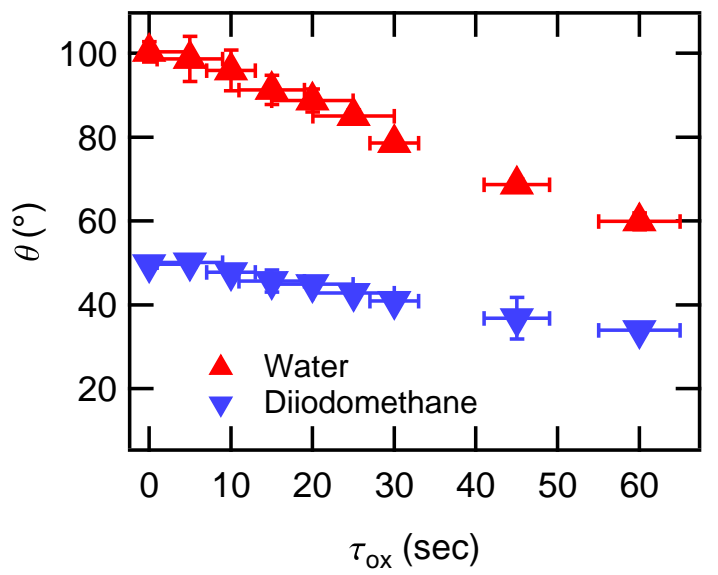

a)

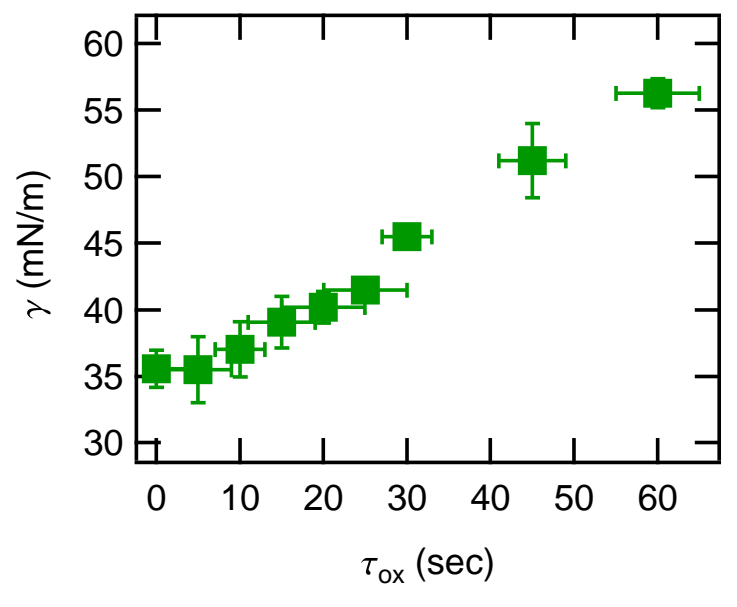

Figure 1: a) Contact angle of water and diiodomethane $(\theta)$ on ODS/silicon substrates as a function of oxidation time. b) Energy of ODS/silicon substrates $(\gamma)$ as a function of oxidation time.

substrates and then annealed at $240{ }^{\circ} \mathrm{C}$ for $10 \mathrm{~min}$ in air. This temperature drives a rapid selfassembly process, and also creates a "neutral" air interface by suppressing the formation of PS wetting layers. ${ }^{7,13,27,28}$ There is no evidence of PS-PMMA degradation after this short-time, hightemperature annealing process. ${ }^{13}$ The variables for these studies were ODS oxidation time $\tau_{o x}(10$ $30 \mathrm{sec}$ ) and normalized film thickness $t / L_{0}(0.5-3)$, where $L_{0}=46 \mathrm{~nm}$ is the equilibrium lamellar periodicity. When oxidation times were less than $15 \sec \left(\theta_{w}>90^{\circ}\right)$, the substrates were difficult to coat with the block copolymer because their surface energies were too low. When oxidation times were greater than $20 \sec \left(\theta_{w}<87^{\circ}\right)$, the lamellar films were optically rough and characterized by a high area fraction of parallel lamellae (Supporting Information Figure S3). Therefore, the 
remainder of this discussion focuses on ODS/silicon substrates that were oxidized for 15 sec or 20 sec, producing water contact angles of $(90 \pm 1)^{\circ}$ and $(87 \pm 1)^{\circ}$, respectively. The corresponding substrate energies are $(39 \pm 1) \mathrm{mN} / \mathrm{m}$ for $15 \mathrm{sec}$ oxidation and $(40 \pm 1) \mathrm{mN} / \mathrm{m}$ for $20 \mathrm{sec}$ oxidation. The substrate energy increases by approximately $3-4 \mathrm{mN} / \mathrm{m}$ during annealing, which is discussed in further detail in the Supporting Information. In the following discussion, we refer to the initial state of the substrate, as this likely templates the domain orientations through the film thickness.

The lamellar domain orientations at the air interface were characterized with AFM and/or SEM. All samples exhibited the classic fingerprint pattern that is characteristic of perpendicular domain orientations, but many had regions of featureless "patches" that arise from parallel layers or highly tilted domains. The area fraction of these patches $\left(f_{\text {patch }}\right)$ was quantified using ImageJ software as illustrated in Figure 2a, and then the area fraction of perpendicular lamellae was calculated by $f_{\text {perp }}=1-f_{\text {patch }}$. Figure 3 summarizes $f_{\text {perp }}$ as a function of normalized film thickness $t / L_{0}$ for the oxidized ODS surfaces. Each data point reports the average of multiple measurements from a single sample and error bars reflect the standard deviation. The dashed line at $f_{\text {perp }}=$ 0.95 marks the transition from perpendicular domains $\left(f_{\text {perp }} \approx 1\right)$ to mixed domain orientations $\left(f_{\text {perp }}<0.95\right)$. This threshold was determined by visual inspection of the data, as imaging artifacts are sometimes confused with patches by the software. Samples with identical symbols were cut from the same oxidized wafer, and open symbols are used for data that fall below the dashed line ("patchy samples"). The same symbols are used throughout the manuscript to facilitate comparison of different results.

With the lower-energy surfaces $\left(\theta_{w} \approx 90^{\circ}\right)$, perpendicular domain orientations were detected at the air interface for film thicknesses of $t / L_{0} \approx 0.7,1.5,1.8,2.3$ and 2.5. Mixed domain orientations were detected at the air interface for all other film thicknesses, including $t / L_{0} \approx$ $0.5,1,1.25,1.5,2$ and 2.75. The apparent coexistence of perpendicular and mixed domain orientations at $t / L_{0} \approx 1.5$ most likely reflects experimental error in the oxidation process, as these data points came from two different wafers. With the higher-energy surfaces $\left(\theta_{w} \approx 87^{\circ}\right)$, the domain orientations at the air interface were perpendicular in all cases where $t / L_{0}>0.5$. 
a)

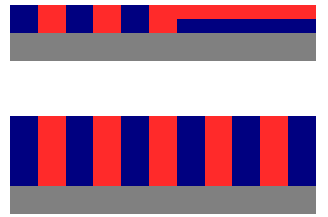

b)
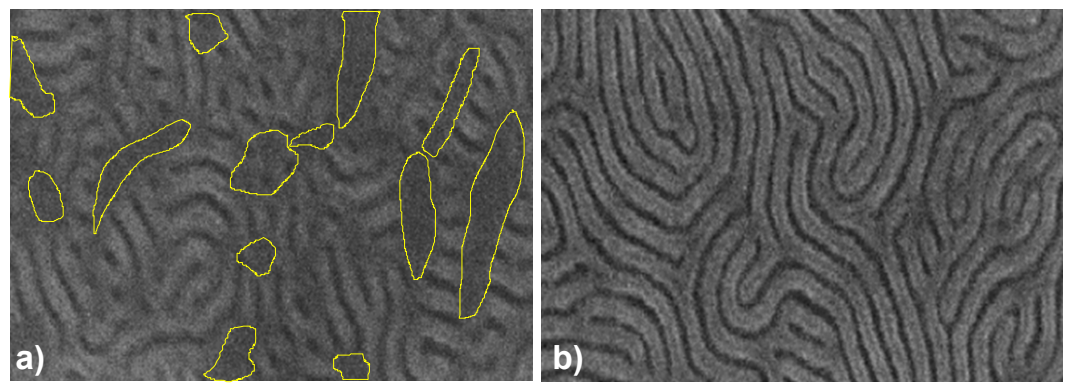

c)

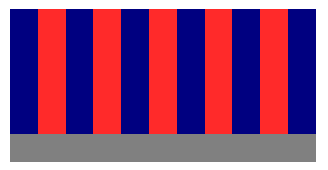

d)
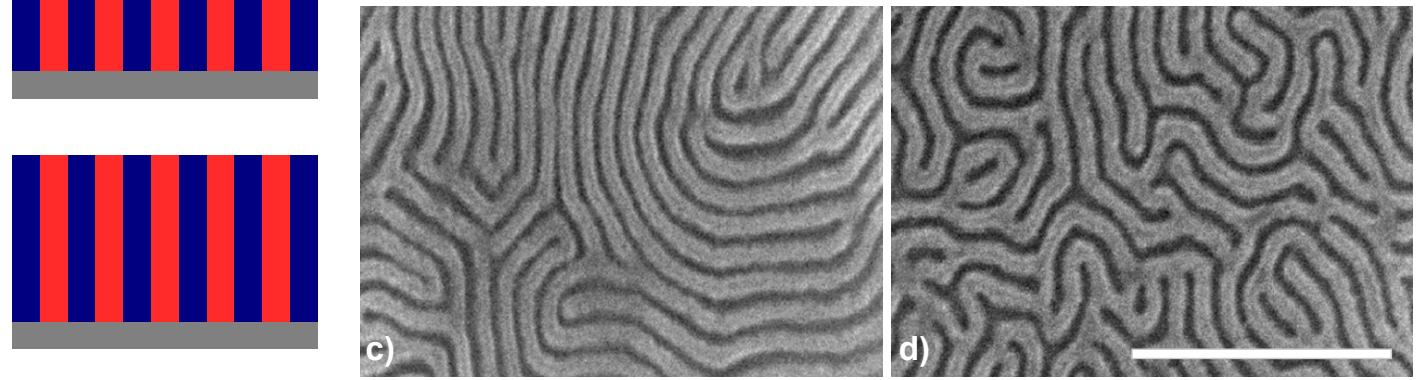

Figure 2: Examples of SEM measurements and idealized cartoons of the cross-section for films cast on oxidized ODS surfaces $\left(\theta_{w} \approx 87^{\circ}\right)$, with normalized thicknesses $t / L_{0}$ of a) 0.5 ; b) 1.25 ; c) 2.3; and d) 2.9. Each image is a subset of a larger data set. The yellow lines in part a) enclose featureless regions that indicate the presence of a surface wetting layer. Scale bar $=500 \mathrm{~nm}$.

The dependence of domain orientations on film thickness usually indicates that one block (either PS or PMMA) is weakly preferred at one or both interfaces. ${ }^{7,13}$ In these experiments, the elevated processing temperature eliminates preferential interactions at the free surface. ${ }^{7,13,27,28}$ The initial ODS surface is extremely low energy, and oxidation introduces oxygen-containing moieties that attract the more polar PMMA blocks. Therefore, it is likely that the oxidized ODS surfaces with $\theta_{w} \approx 90^{\circ}$ are weakly attractive to PS, but the more hydrophilic surfaces with $\theta_{w} \approx 87^{\circ}$ are (approximately) neutral.

In general, when lamellar copolymers are confined between weakly preferential interfaces, certain film thicknesses will impose a condition of high entropic frustration that stabilizes the perpendicular domain orientation. ${ }^{13}$ If the substrate with $\theta_{w} \approx 90^{\circ}$ is weakly preferential to PS, then the conditions of maximum frustration are achieved with as-cast film thicknesses of $t / L_{0}=(n \pm 0.25),{ }^{7}$ so perpendicular domains are anticipated near $t / L_{0} \approx 0.75,1.25,1.75$, etc. This expectation is only partly consistent with the observed data, as perpendicular domains are also observed near $t / L_{0} \approx 1.5$ and 2.5. The origin of this usual behavior is unclear. However, 


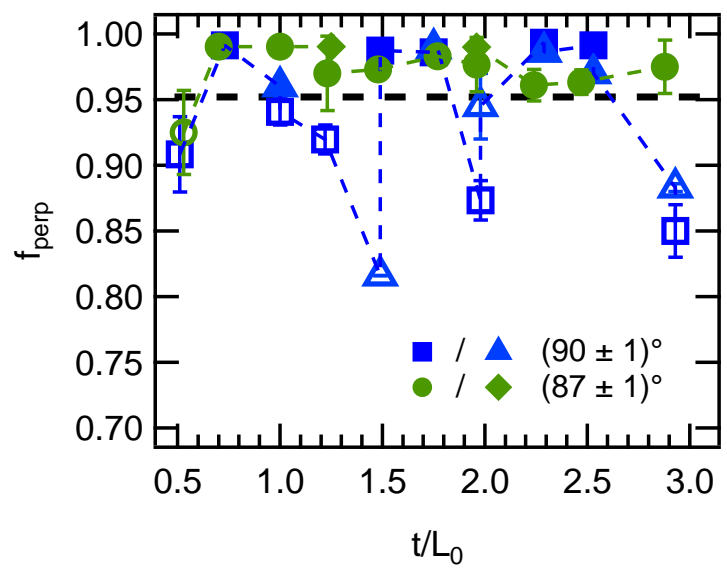

Figure 3: Area fraction of perpendicular lamellar domains $\left(f_{\text {perp }}\right)$ as a function of normalized film thickness $\left(t / L_{0}\right)$ for oxidized ODS surfaces. The legend reports the water contact angle. Samples with identical symbols were prepared from the same oxidized wafer. Horizontal dashed line marks $f_{\text {perp }}=0.95$, and open symbols designate data with $f_{\text {perp }} \leq 0.95$.

oxidation processes are routinely employed to enhance adhesive interactions between polymers and surfaces, ${ }^{29}$ so secondary interactions between PMMA segments and polar moieties could be hindering polymer mobility ${ }^{30-32}$ and trapping unstable structures in the film. Alternatively, confinement near a hard substrate boundary may be frustrating the system to a greater extent than observed with other types of neutral coatings, such as random copolymer brushes.

If interactions or frustration at the oxidized ODS substrate are trapping structures in the film, then such behavior could impact lateral domain order by suppressing the kinetics of defect annihilation. To test for such behavior, lateral order at the free surface was quantified by calculating the orientational correlation function $g(\vec{r})$ for each AFM or SEM image and fitting the azimuthal average to an exponential decay, i.e., $g(r)=\exp (-r / \zeta)$. The exact algorithm is described elsewhere. ${ }^{33,34}$ Average values of the orientational correlation length $\zeta$ were computed from 3-5 micrographs (each spanning at least $2 \mu \mathrm{m} \times 2 \mu \mathrm{m}$ ) and error bars denote the standard deviation. Representative SEM micrographs are included in Fig. 2 for ODS surfaces with $\theta_{w} \approx 87^{\circ}$, and visual inspection reveals clear differences in lateral ordering as a function of film thickness. The outcome $\zeta / L_{0}$ is reported for each ODS surface as a function of normalized film thickness in Fig. 4, where larger values indicate better lateral order (lower defectivity). 
Many studies have characterized the effects of film thickness on lamellar ordering through analysis of orientational correlation lengths, persistence lengths along the lamellar axis, or approximate grain sizes. These works demonstrate that ordering improves as $t^{n}$, where $n$ ranges from 0.4-1. ${ }^{14,35-37}$ The data for low energy ODS surfaces $\left(\theta_{w} \approx 90^{\circ}\right)$ are consistent with this power-law behavior, and the dashed blue line in Fig. 4 marks the trend for $n=0.9$. However, the data for the higher-energy ODS surfaces $\left(\theta_{w} \approx 87^{\circ}\right)$ do not follow the simple power law, but appear to exhibit a maximum near $t / L_{0} \approx(2 \pm 0.3)$. (Alternatively, if the last data point near $t / L_{0} \approx 3$ is neglected, then a power law with exponent $n \approx 1.25$ can roughly describe the trend for $\theta_{w} \approx 87^{\circ}$.) As in the case of $f_{\text {perp }}$ analysis, it seems that oxidation of ODS leads to complex trends with film thickness that are not encountered with other types of neutral (or nearly-neutral) surfaces. The large value of $n$, along with the extremely low value of $\zeta$ in ultrathin films, suggest that strong interactions at the substrate are trapping defects in the film. ${ }^{14}$ Lateral ordering improves with increasing film thickness because the influence of the substrate is reduced, and additionally, there is a stronger energetic drive for defect annihilation in thick films compared with thin films. ${ }^{37}$

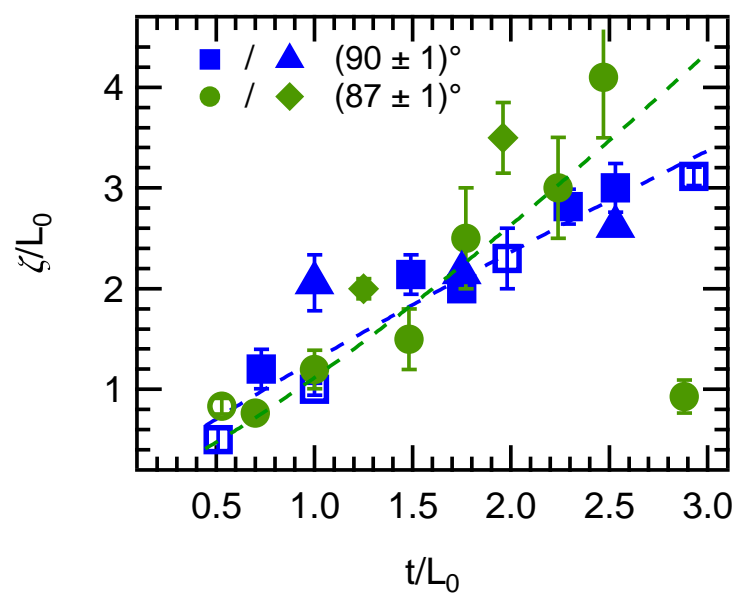

Figure 4: Orientational correlation length at the free surface $\left(\zeta / L_{0}\right)$ as a function of normalized film thickness $\left(t / L_{0}\right)$ on oxidized ODS surfaces. Samples with identical symbols were prepared from the same oxidized wafer. Open symbols designate data with $f_{\text {perp }} \leq 0.95$.

To better understand how substrate interactions impact ordering, we examined domain orientations throughout the film thickness using grazing-incidence small angle X-ray scattering 
(GISAXS). Examples of GISAXS patterns for film thicknesses in the range of $t / L_{0}=1-2$ are included in Fig. 5. The GISAXS intensity (a.u.) is reported as a function of in-plane and out-ofplane scattering angles $2 \Theta$ and $\alpha_{f}$, respectively, and depends on the angle of incidence $\alpha_{i}$. Samples were measured with $\alpha_{i}$ of $0.2^{\circ}, 0.22^{\circ}$, and $0.24^{\circ}$, which are all larger than the polymer's critical angle (ca. $0.17^{\circ}$ ), and data were analyzed through two methods: First, we examined the spectra to see if scattering is consistent with perpendicular lamellae, or a mixture of parallel and perpendicular lamellae. Perpendicular lamellae will produce Bragg reflections along the in-plane axis at positions $2 \Theta=n \lambda / L_{0}$, where $n$ is a positive integer. Parallel lamellae will produce Bragg reflections along the out-of-plane axis; the peak positions along $\alpha_{f}$ depend on refractive index, $L_{0}$, and $\alpha_{i}$. We do not observe the even-order Bragg reflections from either orientation because they coincide with minima in the form factor for equal-volume PS and PMMA domains. ${ }^{38}$ Fig. 5 shows that in-plane (10) and (30) peaks from perpendicular lamellae are observed in all frames. However, the (01) and (03) Bragg peaks from parallel lamellae are only observed in a few data sets which are marked by the " $x$ " and "o" (centered behind the beam stop at $2 \Theta=0$ ). A comprehensive discussion of outof-plane peak indexing in GISAXS can be found elsewhere. ${ }^{39,40}$ Second, we calculate the extent of disorder in the perpendicular phase by analyzing the trajectory of partial Debye-Scherrer rings, which are shown as white curves that superimpose data in Fig. 5. ${ }^{13,14,41}$ Disorder is quantified by the maximum mis-orientation angle $\phi$, where $\phi=0^{\circ}$ indicates a perfect perpendicular orientation, and $\phi=p^{\circ}$ includes all domain orientations in the range of 0 to $p^{\circ} \cdot{ }^{13,14}$

Figure 6 summarizes the outcomes of GISAXS data analysis. Circles and squares designate the maximum mis-orientation angle of a function of film thickness; as before, open and closed symbols reflect samples with $f_{\text {perp }}<0.95$ or $f_{\text {perp }} \geq 0.95$, respectively, based on surface microscopy. Tilt could not be identified in samples with $t / L_{0} \leq 1$, but these samples have broad line shapes due to poor lateral order, so the partial Debye-Scherrer rings might be obscured (resolution limit). ${ }^{14}$ In thicker films, the perpendicular phase is characterized by large misorientation angles up to approximately $40^{\circ}$, so the idealized cartoons inferred from surface microscopy in Fig. 2 are incorrect: the domains are actually bending or tilting through the thickness of the film. Such deformations 

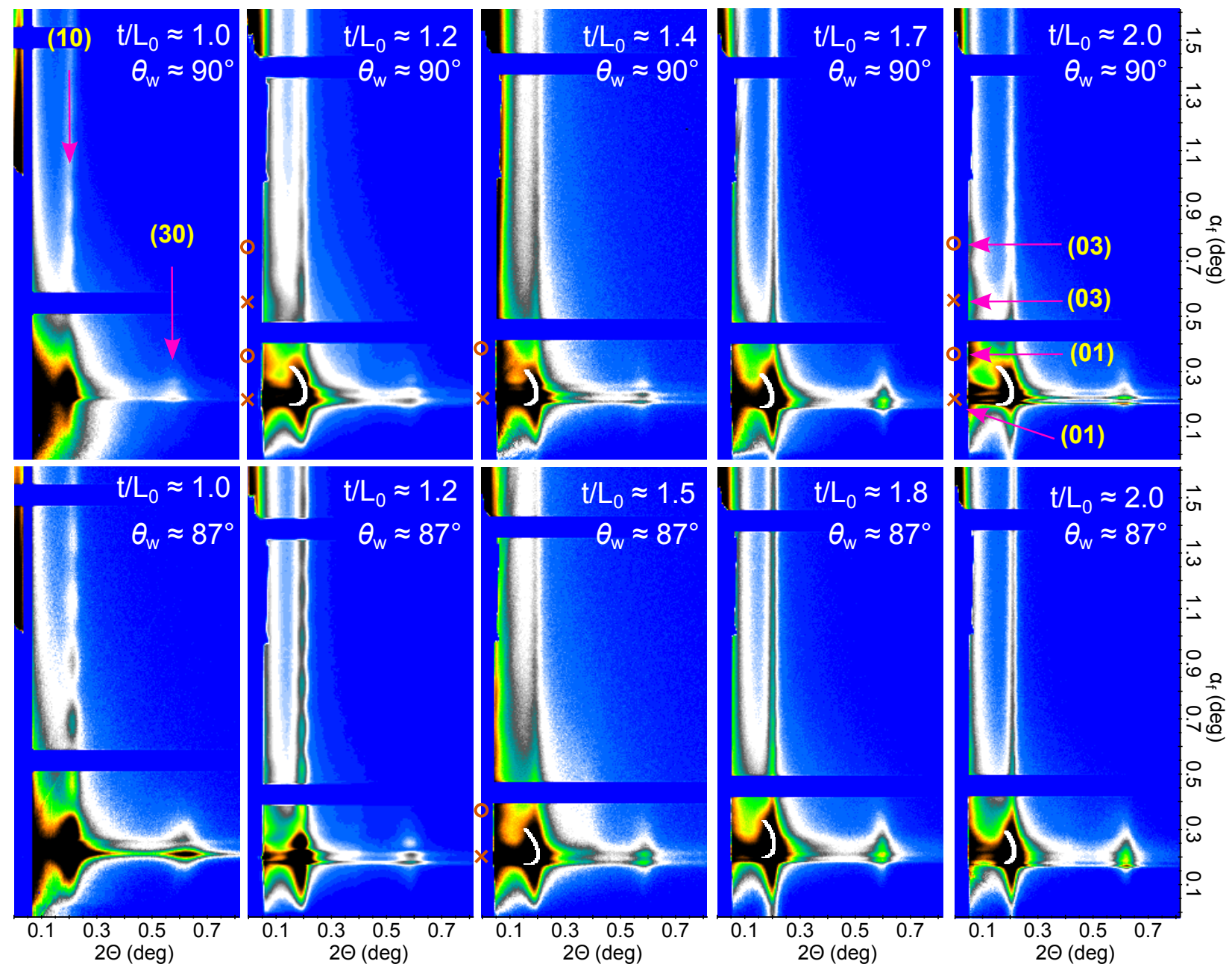

Figure 5: Selected examples of GISAXS data for $\alpha_{i} \approx 0.2^{\circ}$.

could create challenges for pattern transfer in a lithographic process by introducing variability in feature size and placement. ${ }^{42}$ We note that the energetic penalty for bending a soft lamellar domain is very low, so suppressing these defects would require an external guide such as an epitaxial template. ${ }^{14,43}$

Coexistence between perpendicular and parallel domain orientations is clearly detected in GISAXS patterns from some of the samples, and this occurrence is designated by the " $\mathrm{x}$ " and “+” at $\phi=90^{\circ}$ for $\theta_{w} \approx 90^{\circ}$ and $87^{\circ}$, respectively. There are only a few apparent discrepancies between GISAXS and microscopy analysis of domain orientations: First, when $t / L_{0} \approx 0.5$, there is no sign of parallel lamellae in GISAXS patterns for either surface, although featureless patches were 
detected in microscopy $\left(f_{\text {perp }} \approx 0.9\right)$. However, films of this thickness cannot accommodate a full unit cell of parallel lamellae, so scattering from these structures is not anticipated. Second, there are two points where surface microscopy suggested a uniform perpendicular phase $\left(f_{\text {perp }} \geq 0.95\right)$, but GISAXS detects coexistence with parallel lamellae: $t / L_{0} \approx 2.5$ for $\theta_{w} \approx 90^{\circ}$, and $t / L_{0} \approx 1.5$ for $\theta_{w} \approx 87^{\circ}$. This outcome suggests that film structures are not uniform across the entire area of a chip, which is difficult to detect through local measurements such as high resolution microscopy.

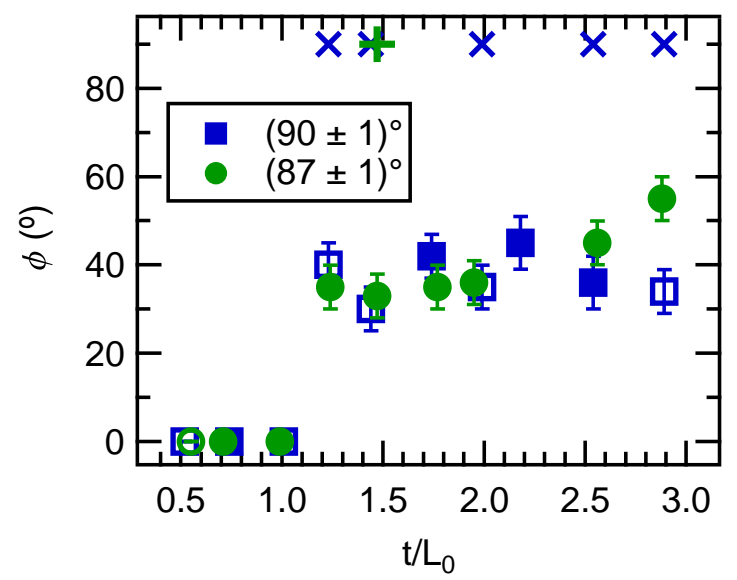

Figure 6: Maximum mis-orientation angle $\phi$ as a function of normalized film thickness (squares and circles). "x" $\left(\theta_{w} \approx 90^{\circ}\right)$ and " $+"\left(\theta_{w} \approx 87^{\circ}\right)$ designate samples that exhibit coexistence between parallel lamellae and disordered perpendicular lamellae.

Comparing the data in Fig. 6 and Fig. 4, it is interesting to note that there is little correlation between out-of-plane and in-plane disorder. The out-of-plane disorder captured by $\phi$ is nearly invariant with film thickness over the range $t / L_{0} \approx 1.25-2.5$, exhibiting a plateau near $\phi \approx 40^{\circ}$ for both oxidized ODS surfaces, while the in-plane disorder inferred from $\zeta$ is strongly suppressed by increasing the film thickness. Furthermore, out-of-plane disorder is not detected in the thinnest films $\left(t / L_{0} \leq 1\right)$, although this may be resolution limited, while lateral disorder is extremely high in these samples (very low $\zeta$ ). Similar trends were observed in our previous work with PS-PMMA lamellae on random copolymer brushes. ${ }^{14}$ These outcomes suggest that surface imaging does not provide a complete picture of lamellar self-assembly throughout the film thickness, but powerful complementary techniques like scattering can help to reveal the three-dimensional structure. ${ }^{44}$ 


\section{Conclusions}

We examined the self-assembly of PS-PMMA lamellae on neutral and nearly-neutral substrates prepared by oxidation of a hydrophobic self-assembled monolayer. This approach offers a simple approach to tune surface energies and stabilize the perpendicular domain orientation over a broad range of film thickness, demonstrating that the substrate can be very close to neutral with an optimized oxidation process. However, the lateral order (captured by orientational correlation length) exhibits a very strong dependence on film thickness, which suggests that secondary interactions at the substrate may be pinning PMMA domains and suppressing the rates of defect annihilation. Furthermore, the out-of-plane disorder in these systems is quite high when the film thickness exceeds one lamellar period, and this may present challenges for high quality pattern transfer in a lithographic process.

\section{Acknowledgement}

The authors acknowledge financial support from the National Science Foundation under Grant No. DMR-1151468. Use of the Advanced Photon Source, an Office of Science User Facility operated for the U.S. Department of Energy (DOE) by Argonne National Laboratory, was supported by the U.S. DOE under Contract No. DE-AC02-06CH11357. The authors thank Dr. Long Chang for assistance with SEM and Dr. Matt Hammond for sharing $g(\vec{r})$ code.

\section{References}

(1) Diederichsen, K. M.; Brow, R. R.; Stoykovich, M. P. ACS Nano 2015, 9, 2465-2476.

(2) Rasappa, S.; Ghoshal, T.; Borah, D.; Senthamaraikannan, R.; Holmes, J. D.; Morris, M. A. Scientific Reports 2015, 5.

(3) Bates, C. M.; Maher, M. J.; Janes, D. W.; Ellison, C. J.; Willson, C. G. Macromolecules 2014, 47, 2-12. 
(4) Morris, M. A. Microelectronic Engineering 2015, 132, 207-217.

(5) Bang, J.; Jeong, U.; Ryu, D. Y.; Russell, T. P.; Hawker, C. J. Advanced Materials 2009, 21, 4769-4792.

(6) Bates, C. M.; Seshimo, T.; Maher, M. J.; Durand, W. J.; Cushen, J. D.; Dean, L. M.; Blachut, G.; Ellison, C. J.; Willson, C. G. Science 2012, 338, 775-779.

(7) Kim, S.; Bates, C. M.; Thio, A.; Cushen, J. D.; Ellison, C. J.; Willson, C. G.; Bates, F. S. ACS Nano 2013, 7, 9905-9919.

(8) Ryu, D.; Shin, K.; Drockenmuller, E.; Hawker, C.; Russell, T. Science 2005, 308, 236-239.

(9) Mansky, P.; Liu, Y.; Huang, E.; Russell, T. P.; Hawker, C. J. Science 1997, 275, 1458-1460.

(10) Han, E.; Stuen, K. O.; La, Y.-H.; Nealey, P. F.; Gopalan, P. Macromolecules 2008, 41, 90909097.

(11) Kim, M.; Han, E.; Sweat, D. P.; Gopalan, P. Soft Matter 2013, 9, 6135-6141.

(12) Sparnacci, K.; Antonioli, D.; Gianotti, V.; Laus, M.; Ferrarese Lupi, F.; Giammaria, T. J.; Seguini, G.; Perego, M. ACS Applied Materials \& Interfaces 2015, 7, 10944-10951.

(13) Vu, T.; Mahadevapuram, N.; Perera, G. M.; Stein, G. E. Macromolecules 2011, 44, 61216127.

(14) Mahadevapuram, N.; Mitra, I.; Bozhchenko, A.; Strzalka, J.; Stein, G. E. Journal of Polymer Science Part B: Polymer Physics 2015, n/a-n/a.

(15) Guo, R.; Kim, E.; Gong, J.; Choi, S.; Ham, S.; Ryu, D. Y. Soft Matter 2011, 7, 6920-6925.

(16) Onclin, S.; Ravoo, B. J.; Reinhoudt, D. N. Angewandte Chemie International Edition 2005, 44, 6282-6304. 
(17) Albert, J. N. L.; Baney, M. J.; Stafford, C. M.; Kelly, J. Y.; Epps, T. H. ACS Nano 2009, 3, 3977-3986.

(18) Albert, J. N.; Kim, J. D.; Stafford, C. M.; Epps III, T. H. Review of Scientific Instruments 2011, 82, 065103.

(19) Luo, M.; Seppala, J. E.; Albert, J. N. L.; Lewis, R. L.; Mahadevapuram, N.; Stein, G. E.; Epps, T. H. Macromolecules 2013, 46, 1803-1811.

(20) Kim, S. O.; Solak, H. H.; Stoykovich, M. P.; Ferrier, N. J.; de Pablo, J. J.; Nealey, P. F. Nature 2003, 424, 411-414.

(21) Liu, P.-H.; Thébault, P.; Guenoun, P.; Daillant, J. Macromolecules 2009, 42, 9609-9612.

(22) Zhao, Y.; Sivaniah, E.; Hashimoto, T. Macromolecules 2008, 41, 9948-9951.

(23) Wu, S. Journal of Polymer Science Part C: Polymer Symposia 1971, 34, 19.

(24) Wu, S. J. Adhesion 1973, 5, 39-55.

(25) Wu, S. The Journal of Physical Chemistry 1970, 74, 632-638.

(26) Dann, J. Journal of Colloid and Interface Science 1970, 32, 302-320.

(27) Mansky, P.; Russell, T. P.; Hawker, C. J.; Mays, J.; Cook, D. C.; Satija, S. K. Physical Review Letters 1997, 79, 237-240.

(28) Han, E.; Stuen, K. O.; Leolukman, M.; Liu, C.-C.; Nealey, P. F.; Gopalan, P. Macromolecules 2009, 42, 4896-4901.

(29) Chan, C. M.; Ko, T. M.; Hiraoka, H. Surface Science Reports 1996, 24, 1-54.

(30) Keddie, J. L.; Jones, R. A. L.; Cory, R. A. Faraday Discussions 1994, 98, 219-230.

(31) Fryer, D.; Peters, R.; Kim, E.; Tomaszewski, J.; de Pablo, J.; Nealey, P.; White, C.; Wu, W. Macromolecules 2001, 34, 5627-5634. 
(32) Tate, R.; Fryer, D.; Pasqualini, S.; Montague, M.; de Pablo, J.; Nealey, P. Journal OfChemical Physics 2001, 115, 9982-9990.

(33) Harrison, C.; Adamson, D. H.; Cheng, Z.; Sebastian, J. M.; Sethuraman, S.; Huse, D. A.; Register, R. A.; Chaikin, P. M. Science 2000, 290, 1558-1560.

(34) Hammond, M. R.; Cochran, E.; Fredrickson, G. H.; Kramer, E. J. Macromolecules 2005, 38, $6575-6585$.

(35) Park, S.-M.; Berry, B. C.; Dobisz, E.; Kim, H.-C. Soft Matter 2009, 5, 957-961.

(36) Sivaniah, E.; Hayashi, Y.; Matsubara, S.; Kiyono, S.; Hashimoto, T.; Fukunaga, K.; Kramer, E. J.; Mates, T. Macromolecules 2005, 38, 1837-1849.

(37) Campbell, I. P.; Hirokawa, S.; Stoykovich, M. P. Macromolecules 2013, 46, 9599-9608.

(38) Renaud, G.; Lazzari, R.; Leroy, F. Surface Science Reports 2009, 64, 255-380.

(39) Stein, G. E.; Kramer, E. J.; Li, X.; Wang, J. Macromolecules 2007, 40, 2453-2460.

(40) Vogt, B. D.; Chavez, V. L.; Dai, M.; Arreola, M. R. C.; Song, L.; Feng, D.; Zhao, D.; Perera, G. M.; Stein, G. E. Langmuir 2011, 27, 5607-5615.

(41) Mueller-Buschbaum, P.; Maurer, E.; Bauer, E.; Cubitt, R. Langmuir 2006, 22, 9295-9303.

(42) Bosworth, J. K.; Dobisz, E. A.; Hellwig, O.; Ruiz, R. Macromolecules 2011, 44, 9196-9204.

(43) Perera, G. M.; Wang, C.; Doxastakis, M.; Kline, R. J.; Wu, W.-1.; Bosse, A. W.; Stein, G. E. Acs Macro Letters 2012, 1, 1244-1248.

(44) Stein, G. E.; Mahadevapuram, N.; Mitra, I. Journal of Polymer Science Part B: Polymer Physics 2015, 53, 96-102. 


\section{Ordering of Lamellar Block Copolymers on Oxidized Silane Coatings}

Nikhila Mahadevapuram, Indranil Mitra, Shyam Sridhar, Joseph Strzalka, Gila E. Stein

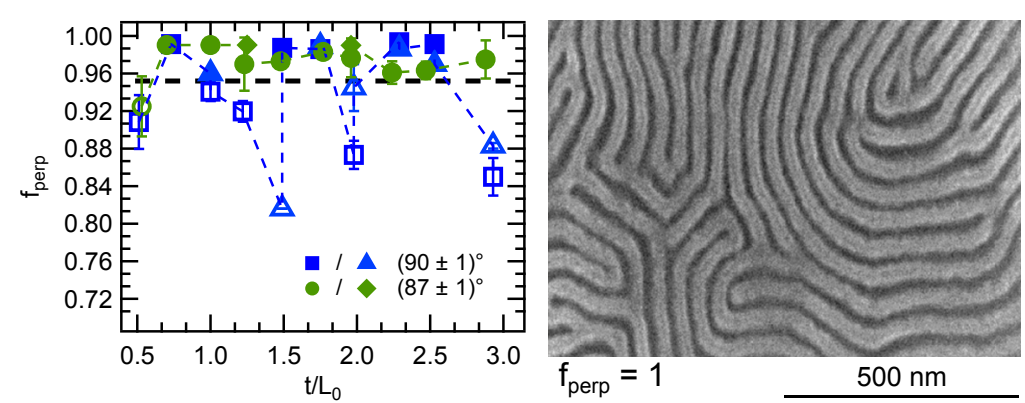

